\title{
Crystal Structure of the Siderophore Binding Protein BauB Bound to an Unusual 2:1 Complex Between Acinetobactin and Ferric Iron
}

\author{
Daniel C. Bailey ${ }^{1}$, Tabbetha J. Bohac ${ }^{2}$, Justin A. Shapiro², Daryl E. Giblin², Timothy A. \\ Wencewicz ${ }^{2,}$, and Andrew M. Gulick ${ }^{*}, 1$ \\ ${ }^{1}$ Department of Structural Biology, Jacobs School of Medicine \& Biomedical Sciences at the \\ University at Buffalo, 955 Main St., Buffalo, NY, 14203 USA. \\ ${ }^{2}$ Department of Chemistry, Washington University in St. Louis, One Brookings Dr, St. Louis, MO, \\ 63130 USA
}

\begin{abstract}
The critical role that iron plays in many biochemical processes has led to an elaborate battle between bacterial pathogens and their hosts to acquire and withhold this critical nutrient. Exploitation of iron nutritional immunity is being increasingly appreciated as a potential antivirulence therapeutic strategy, especially against problematic multi-drug resistant Gramnegative pathogens such as Acinetobacter baumannii. To facilitate iron uptake and promote growth, $A$. baumannii produces a nonribosomally synthesized peptide siderophore called acinetobactin. Acinetobactin is unusual in that it is first biosynthesized in an oxazoline form called pre-acinetobactin that spontaneously isomerizes to the final isoxazolidinone acinetobactin. Interestingly, both isomers can bind iron and both support growth of $A$. baumannii. To address how the two isomers chelate their ferric cargo and how the complexes are used by $A$. baumannii, structural studies were carried out with the ferric acinetobactin complex and its periplasmic siderophore binding protein BauB. Herein, we present the crystal structure of BauB bound to a bis-tridentate $\left(\mathrm{Fe}^{3+} \mathrm{L}_{2}\right)$ siderophore complex. Additionally, we present binding studies that show multiple variants of acinetobactin bind BauB with no apparent change in affinity. These results are consistent with the structural model that depicts few direct polar interactions between BauB and the acinetobactin backbone. This structural and functional characterization of acinetobactin and its requisite binding protein $\mathrm{BauB}$ provides insight that could be exploited to target this critical iron acquisition system and provide a novel approach to treat infections caused by this important multidrug resistant pathogen.
\end{abstract}

\section{Graphical Abstract}

*To whom correspondence should be addressed:AMG: amgulick@buffalo.edu; Ph 716-829-3696, TAW: wencewicz@wustl.edu; Ph: 314-935-7247; Fax: 314-935-4481.

Supporting Information. The supporting information is available free of charge on the ACS Publications website at DOI: 


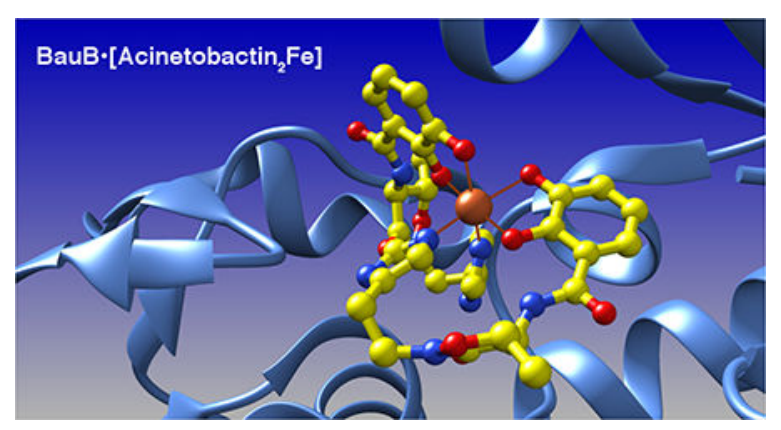

\section{Keywords}

Acinetobacter baumannii; siderophore; iron transport; acinetobactin; siderophore binding protein

\section{INTRODUCTION}

To cope with the challenges faced in many environments with scarce bioavailable iron, many bacteria produce siderophores, small molecule iron chelators that are secreted into the environment where they form ferric complexes that are transported back into the cell. ${ }^{1,2}$ The requirement for iron in almost all forms of life has motivated efforts to understand the production and utilization of hundreds of distinct siderophores, including the diversity of chemical structures and the ability of bacteria to use siderophores made by other species. Of particular interest, siderophores have been shown to play an important role in many pathogenic environments due to the limiting concentration of iron within the host. This raises the potential to target siderophore biosynthesis, transport, or uptake as a novel approach to limit the establishment or worsening of a bacterial infection. ${ }^{3-5}$

Siderophores use several key binding motifs to interact with iron, the most common being phenol and catechol oxygens, as well as hydroxamates, and carboxylate moieties. ${ }^{1}$ There are two broad classes of siderophores, those produced by the nonribosomal peptide synthetases (NRPSs) ${ }^{6,7}$ and a more recently discovered family of NRPS-independent siderophores (NISs). ${ }^{5,8,9}$ Both enzyme systems use a wide array of building blocks in their biosynthetic pathways. The NRPSs combine proteinogenic and more specialized amino acids, aromatic acids, and short- to long-chain fatty acids via a modular enzymatic architecture. ${ }^{7}$ In contrast, NIS pathways attach catechol, carboxylate, and hydroxamate building blocks to a polyamine or citrate backbone to form metal chelating compounds.

After export of the siderophore and subsequent iron binding, the ferric siderophore must then be taken back into the cell. In Gram-negative bacteria, siderophore uptake is initiated by a siderophore-selective TonB outer membrane receptor. ${ }^{10}$ Once transported into the periplasm, the siderophore is bound by a siderophore binding protein (SBP) that mediates the transfer of the siderophore to an ATP-binding cassette (ABC) transporter for delivery across the inner membrane and into the cytoplasm. ${ }^{11-13} \mathrm{ABC}$ transporters are transmembrane proteins that are involved in the import and export of a wide variety of molecules. Their cytoplasmic nucleotide binding domains catalyze ATP hydrolysis to drive conformational changes of the transmembrane domain that enable translocation of the 
ligand. $\mathrm{ABC}$ transporters involved in ligand import interact with a variety of substrate binding proteins that capture the ligand in the periplasm. These transfer partners can either be soluble periplasmic proteins, tethered to the inner membrane through post-translational lipidation, or indeed tethered directly to the $\mathrm{ABC}$ transporter. ${ }^{11,14}$

SBPs belong to a larger family of binding proteins that play many roles in transport and uptake of metals, amino acids, and peptides. ${ }^{15,16}$ These proteins contain two small $\alpha / \beta$ domains, each with a central $\beta$-sheet that is surrounded by $\alpha$-helices. The ligand binding pocket is located between the two globular domains forming a cradle-like structure. In many members of the family, the relative orientation of the two domains is flexible, resulting from a hinge motion that accompanies substrate binding. A recently updated analysis identifies seven clusters of substrate binding proteins, with the strructural motif that spans the two $\alpha / \beta$ domains forming a distinguishing feature between different groups. SBPs belong to Cluster $\mathrm{A}$, in which a central $a$-helix forms the bridge between the two smaller domains. ${ }^{16}$ This relatively rigid helix limits the hinge motion between the $\alpha / \beta$ domains and ligand-free and ligand-bound structures adopt very similar conformations regardless of ligand content. ${ }^{11}$

The human pathogen Acinetobacter baumannii produces three siderophores. Two siderophores, acinetobactin ${ }^{17,18}$ and fimsbactin ${ }^{19}$, are produced through NRPSs, while a third is the NIS baumannoferrin ${ }^{20}$. Of 15 representative $A$. baumannii strains present in the Virulence Factors in Pathogenic Bacteria database ${ }^{21}$, fimsbactin is present in only a single genome (strain ATCC17978). Both acinetobactin 1 and baumanoferrin are present in the other strains. (A. baumannii SDF, a strain that was isolated from a body lice and which shows many unusual features ${ }^{22}$, lacks all three siderophore clusters.) Acinetobactin production has been identified as being an important virulence factor in studies in both insect and murine infection models. ${ }^{23}$ Interestingly, acinetobactin is produced in an oxazoline form known as pre-acinetobactin $\mathbf{2}$ that can spontaneously isomerize into the mature isoxazolidinone (Figure 1). The two forms were found to be favored at acidic and basic $\mathrm{pH}$, respectively, which suggests the single siderophore has evolved for use in multiple environments. ${ }^{24}$

Inhibitors of BasE, an NRPS adenylation domain required for acinetobactin biosynthesis, have been identified by rational design and empiric screening; crystal structures of the most potent inhibitors bound to BasE have been determined. ${ }^{25,}{ }^{26}$ While showing promising biochemical activity, the compounds failed to function with whole cells, likely due to poor cell permeability. An alternative approach to inhibition of siderophore biosynthesis includes targeting ferric-siderophore uptake and transport. A variety of acinetobactin analogs have been synthesized that illustrate that functional groups required for iron binding are similarly important in the ability to support $A$. baumannii growth in low iron conditions. ${ }^{27}$

Additionally, oxidation of the oxazoline ring of preacinetobactin to the oxazole results in an analog that is able to compete in a dose-dependent manner with the ability of acinetobactin to support of $A$. baumannii growth in low iron conditions. ${ }^{28}$ To extend these ongoing studies, we describe herein the liganded structure of the siderophore binding protein BauB from the acinetobactin uptake system. The binding pocket of BauB is populated by a bistridentate ferric acinetobactin complex [( $\left.\mathrm{Acb})_{2} \mathrm{Fe}\right]^{-}$that is stabilized by iron(III)coordinating bonds with catechol oxygen and imidazole nitrogen atoms from acinetobactin. 
This structural model is further supported by functional characterization of

BauB:acinetobactin binding, including dissociation constants for BauB with apo and holo acinetobactin, as well as a panel of acinetobactin structural analogs that probe the key recognition elements required for binding.

\section{EXPERIMENTAL METHODS}

\section{Cloning, Expression, and Purification.}

Multiple protein constructs were designed for use in the structural and functional studies. The 969-bp bauB gene from Acinetobacter baumannii (Genbank Accession Number AAT52185) was first subcloned into a pET24b expression vector using restriction enzymebased cloning. Expression of the full-length protein resulted in most of the protein purifying as soluble aggregate, with a small proportion isolated as folded protein. The soluble protein migrated on analytical gel filtration with an elution volume consistent with a monomer (Figure S1). Analysis of the full-length protein construct (1-322) revealed a putative signal peptide (1-22) at the $N$-terminus directing trafficking to the periplasm ${ }^{29}, 30$.

A second expression construct was designed without the putative signal peptide that encoded the mature protein. Following the starting Met residue, the final expression construct contained BauB residues 23-322 and a $C$-terminal $6 \times$ His tag (...LEHHHHHH). Of note, Cys 23 was predicted to be a $N$-terminal lipidation site 31,32 and the presence of Asp at position 24 suggests that upon cleavage of the signal peptide in the periplasm, the protein is natively retained at the periplasmic surface of the of the cytoplasmic membrane according to the "D+2 Rule" 33,34 .

E. coli BL21(DE3) were transformed with the expression plasmid for protein production. Cells were grown to an $\mathrm{OD}_{600}$ of $0.6-0.8$ at $37^{\circ} \mathrm{C}(250 \mathrm{RPM})$ and induced to express BauB with the addition of $500 \mu \mathrm{M}$ IPTG. Following induction, the cells were incubated at $16^{\circ} \mathrm{C}$ overnight $\left(\approx 18 \mathrm{~h}\right.$ ) before being harvested by centrifugation at $6 \times 10^{3} g$ at $4{ }^{\circ} \mathrm{C}$ for $15 \mathrm{~min}$. The cell pellet was flash frozen in liquid $\mathrm{N}_{2}$ and stored at $-80^{\circ} \mathrm{C}$.

The frozen cell pellet $(\approx 10 \mathrm{~g})$ was resuspended in $100 \mathrm{~mL}$ of lysis buffer $(50 \mathrm{mM}$ Tris, 300 $\mathrm{mM} \mathrm{NaCl}, 0.2 \mathrm{mM}$ TCEP, $5 \%$ glycerol, $20 \mathrm{mM}$ imidazole, $\mathrm{pH}$ 8.0) containing protease inhibitor cocktail, DNAse $(10 \mu \mathrm{g} / \mathrm{mL})$, and lysozyme $(1 \mathrm{mg} / \mathrm{mL})$ and incubated at $4{ }^{\circ} \mathrm{C}$ for $30 \mathrm{~min}$. Cell lysis was further ensured by sonication $(5 \times 30 \mathrm{~s}$ cycles $)$. The cell slurry was separated by ultra-centrifugation at $185 \times 10^{3} \mathrm{~g}$ for $40 \mathrm{~min}$ at $4{ }^{\circ} \mathrm{C}$. The supernatant was further clarified via filtration over a $0.45 \mu \mathrm{m}$ polysulfone membrane. The clarified supernatant was subjected to immobilized metal affinity chromatography (IMAC) by passing it over two $5 \mathrm{~mL} \mathrm{Ni}^{2+}$-NTA columns in series. Bound proteins were eluted by passing lysis buffer plus $300 \mathrm{mM}$ imidazole over the columns. To remove imidazole, the protein was dialyzed ( $12 \mathrm{kDa} \mathrm{MWCO}$ ) overnight at $4{ }^{\circ} \mathrm{C}$ in $50 \mathrm{mM}$ Tris, $300 \mathrm{mM} \mathrm{NaCl}, 0.2 \mathrm{mM}$ TCEP, $0.5 \mathrm{mM}$ EDTA, $5 \%$ glycerol, $\mathrm{pH}$ 8.0. The dialyzed protein sample was concentrated using a centrifugal filter (10 kDa MWCO) before carrying out preparative size exclusion chromatography (SEC). Protein was eluted over the SEC column (HiLoad 16/60 Superdex 200, GE Healthcare) using a buffer of $50 \mathrm{mM}$ Tris, $150 \mathrm{mM} \mathrm{NaCl}, 0.2 \mathrm{mM}$ TCEP, $\mathrm{pH} 8.0$ at $1 \mathrm{~mL} / \mathrm{min}$ (Figure S1). The desired fractions containing pure BauB as indicated by SDS- 
PAGE were combined, concentrated to $\approx 60 \mathrm{mg} / \mathrm{mL}$ using a $10 \mathrm{kDa}$ MWCO centrifugal filter, flash frozen in liquid $\mathrm{N}_{2}$, and stored at $-80^{\circ} \mathrm{C}$.

An N-terminally tagged protein, lacking the signal sequence, was also produced and used in binding studies. $N$-His 6 -BauB was expressed from a pET28bTEV plasmid in E. coli BL21 (DE3). Briefly, E. coli BL21 (DE3) transformed with the BauB expression plasmid were grown in terrific Broth at $37^{\circ} \mathrm{C}$ in a $3 \mathrm{~L}$ baffled flask, in the presence of $50 \mu \mathrm{M}$ kanamycin to an $\mathrm{OD}_{600}$ of $\sim 0.5$. The culture flask was cooled to $20^{\circ} \mathrm{C}$ and $\mathrm{BauB}$ expression was induced by the addition of $0.5 \mathrm{mM}$ IPTG (final concentration). Cells were grown overnight $(\sim 12 \mathrm{~h})$ at $20^{\circ} \mathrm{C}$. Cells were harvested via centrifugation at $4{ }^{\circ} \mathrm{C}$ for $30 \mathrm{~min}$ (all remaining steps were carried out at $\left.4{ }^{\circ} \mathrm{C}\right)$. Cells were washed with lysis buffer $(50 \mathrm{mM}$ potassium phosphate $\mathrm{pH}$ 8.0, $500 \mathrm{mM} \mathrm{NaCl}, 5 \mathrm{mM}$ BME, $20 \mathrm{mM}$ imidazole, $10 \%$ glycerol) and flash frozen in liquid $\mathrm{N}_{2}$ ( $40 \mathrm{~mL}$ total volume). The frozen cell pellet was thawed and lysed by two passes through an Emulsiflex C5 (Avestin). The lysate was clarified by ultracentrifugation at $50000 \mathrm{~g}$ for $35 \mathrm{~min}$. The supernatant was passed through Ni-NTA resin and eluted with $300 \mathrm{mM}$ imidazole in lysis buffer. Elution fractions were analyzed by SDSPAGE with visualization by Coomassie blue staining. Fractions containing pure $\mathrm{N}$-His ${ }_{6}^{-}$ BauB were combined, dialyzed (50 mM potassium phosphate $\mathrm{pH} 8.0,150 \mathrm{mM} \mathrm{NaCl}, 1 \mathrm{mM}$ DTT, 5\% glycerol), and concentrated via centrifugal filtration. Protein purity was analyzed by SDS-PAGE with visualization by Coomassie blue staining. Protein identities were confirmed by ESI-MS. Purified protein was flash frozen in liquid $\mathrm{N}_{2}$ at $180 \mu \mathrm{M}$ and stored at $-80{ }^{\circ} \mathrm{C}$. The final protein used in the binding studies contained the $\mathrm{His}_{6}$ tag and TEV cleavage site upstream of $\mathrm{C} 23$ from the BauB sequence.

\section{Crystallization and X-ray diffraction data collection.}

Two protein crystals were used to collect a complete dataset for structure determination. Crystallization and X-ray data collection parameters for both datasets are summarized in Table S1. Briefly, BauB was exchanged into a minimal crystallization buffer and ferric acinetobactin $\left(\mathrm{Acb}_{2} \mathrm{Fe}\right)$ in DMSO was added and incubated on ice for $1 \mathrm{~h}$. Acb was isolated from A. baumannii ATCC 17978 and purified by RP-C18 prep HPLC as described previously. ${ }^{24} \mathrm{Acb}_{2} \mathrm{Fe}$ was prepared by treating Acb with $\mathrm{Fe}(\mathrm{acac})_{3}$ as described previously. 24 The sample was briefly centrifuged to pellet any precipitate before being crystallized by hanging-drop vapor diffusion. Initial crystallization conditions were identified using an inhouse 120-condition screen in which BauB formed thin needle/plate-like crystals in cocktail conditions containing medium to high molecular weight polyethylene glycol (PEG 4K, 8K, or PEG MME 5K) over a relatively wide $\mathrm{pH}(6.0-9.0)$ range. Crystals were flash frozen in liquid $\mathrm{N}_{2}$ and shipped to synchrotron beamlines for remote diffraction data collection.

\section{Diffraction data processing, structure determination, and refinement.}

BauB crystallized in a monoclinic space group $P 2{ }_{1}$ with $\beta=90^{\circ}$. Careful analysis showed that each dataset was not twinned nor could higher symmetry be imposed on the data. Scaling the data in the orthorhombic space group P222 resulted in $R_{\text {merge }}$ values of 0.25 , compared to 0.09 determined with monoclinic space group. The structure of ferric Acb-bound BauB was initially solved by molecular replacement to $1.9 \AA$ resolution employing Data Set 1 (Table S1) using the protein atoms of $\mathrm{YclQ}^{35}$ (PDB 3GFV, 33\% sequence identical) as the 
search model. Due to the thin needle/plate-like morphology of the crystals, it was difficult to achieve high completeness from a single crystal despite collecting images over an oscillation range of $360^{\circ}$. We therefore merged the data from the initial crystal with data from a second crystal. To do this, the two integrated reflection files from MOSFLM were scaled together with AIMLESS of the CCP4 software suite. Although this second dataset was weaker in overall intensity, the increase in completeness was viewed as a reasonable compromise with the resulting poorer merging statistics from combining the two datasets. Processing statistics for the individual (Table S1) and combined (Table 1) data sets are provided. The merged dataset was then used to refine the structure of BauB complexed with $\mathrm{Acb}_{2} \mathrm{Fe}$. All data processing and merging was carried out using iMosflm ${ }^{36}$, molecular replacement employing Phaser $^{37}$, automated model building using PHENIX.Autobuild ${ }^{38}$, manual model building and refinement utilizing $\operatorname{Coot}^{39}$, and automated refinement employing PHENIX.Refine ${ }^{40}$. Structure-based alignments were performed with PROMALS3d. ${ }^{41}$ The atomic coordinates and structure factors for BauB complexed with ferric Acb have been deposited in the PDB (6MFL).

\section{BauB binding studies.}

Acinetobactin (1) and analogs (3-9) were prepared as described previously. ${ }^{27}$ A stock solution of $\mathrm{N}$-His 6 -BauB, thawed on ice, was prepared at $400 \mathrm{nM}$ in assay buffer $(25 \mathrm{mM}$ Tris-HCl, $8 \mathrm{~g} / \mathrm{L} \mathrm{NaCl}, 0.2 \mathrm{~g} / \mathrm{L} \mathrm{KCl}, \mathrm{pH}$ 7.4). For each experiment, $300 \mu \mathrm{L}$ of the BauB stock solution was transferred to a fluorescence cuvette (HellmaAnalytics High Precision Cell cuvette made of Quartz SUPRASIL; light path $10 \times 2 \mathrm{~mm}$ ) in the presence of substrate (compounds 1, 1-Fe, and 3-9) at variable concentrations ranging from 100-1200 nM. An emission spectrum was taken using a PerkinElmer LS 55 Luminescence Spectrometer (slit width $10 \mathrm{~nm}$; scan speed $400 \mathrm{~nm} / \mathrm{min}$ ) at 300-400 $\mathrm{nm}$ using an excitation wavelength of 280 $\mathrm{nm}$. Fluorescence intensity at $320 \mathrm{~nm}$ versus substrate concentration (nM) was plotted. The apparent $K_{\mathrm{d}}$ was calculated by nonlinear regression using a one binding site model in GraphPad Prism v7.0b (Supplementary Information, Figure S7). All experiments were performed in duplicate as independent trials.

\section{DFT Calculations.}

For starting geometry of the bimetallic complexes, we chose the crystal structure of the acinetobactin 2 FeIII $(\mathrm{S}=5 / 2)$ complex bound to BauB. Conformer spaces for complexes were explored by Monte Carlo/MMFF molecular mechanics/dynamics and first step of optimization was performed by using the PM3d semi-empirical algorithm (Spartan Linux v10, WaveFunction, Inc.). Using either as input, we employed DFT (Density Functional Theory, Gaussian 09, Gaussian Inc.) for calculations by using the PBE0 hybrid functional (PBE1PBE in Gaussian parlance) with basis sets Def2-SVP and Def2-TZVP. Minima were optimized at the level PBE0/Def2-SVP and single-point energies were calculated at level PBE0/Def2-TZVP, with scaled thermal-energy corrections from scaling factors for B3LYP/6-31G(d,p). ${ }^{42}$ Solvent-based single-point energies were calculated at the same level by using the CPCM polarizable conductor calculation model for water and using the Universal Force Field for atomic radii. ${ }^{43}$ DFT was selected for computational efficiency and integrity. ${ }^{44,}{ }^{45}$ Functional and basis sets were chosen for use with the ferric complexes. ${ }^{46-48}$ 


\section{RESULTS AND DISCUSSION}

\section{Structure Determination of BauB.}

The structure of BauB was determined by molecular replacement using YclQ, the siderophore binding protein from petrobactin binding in Bacillus subtilis. ${ }^{35}$ The model contains two chains in the asymmetric unit, each of which contains residues 39-322, with a single disordered loop. The two chains do not appear to form a dimeric structure, consistent with the monomeric gel filtration results. In chain A, residues 236-238 are disordered, while in chain B, residues 236-240 are disordered. Additionally, beyond the C-terminal Gln322 residues, each chain contains density for three residues, Leu323-Glu324-His325, derived from the His-tag used for purification. In both chains, the $\mathrm{N}$-terminal 17 residues are also disordered, with Glu39 being the first observed residue. The two chains superimpose with an RMS displacement of $0.15 \AA$ Aver 279 residues (Figure S2).

BauB adopts the classic two domain conformation seen previously with SBPs of the Cluster A family of substrate binding proteins. ${ }^{16}$ The two globular $\alpha / \beta$ domains are bridged by a central helix formed by residues Glu171-Thr192 (Figure 2). Use of the DALI structural similarity server identified multiple homologs within the substrate binding protein family. ${ }^{49}$ The closest homologs, with sequence identities above $25 \%$, all show RMS displacements of $1.9-3.0$ Å over Ca positions (Table S2, Figure S3).

\section{Active Site Contents and Structure of Acinetobactin ${ }_{2} \mathrm{Fe}^{3+}$ complex.}

The siderophore binding pocket of BauB shows clear density for the binding of a complex between two molecules of acinetobactin and a single ferric ion. Each acinetobactin molecule donates both catechol oxygen atoms and one nitrogen atom of the imidazole moiety to form an octahedral arrangement around the central ferric ion. The electron density unambiguously correlates with the molecular structure of the acinetobactin isomer (provided in the crystallization cocktail) and not pre-acinetobactin (which was not provided). This is consistent with the irreversible nature of the pre-acinetobactin to acinetobactin isomerization (Figure S4).

One molecule of acinetobactin is more deeply buried in the substrate binding pocket, while the second is more surface exposed (Figure 3). Specifically, for the buried molecule, only the 2,3-dihydroxybenzoate (DHB) moiety is solvent accessible while the histamine and isoxazolidinone groups are positioned below the ferric ion against the surface of BauB. In contrast, the second acinetobactin molecule lies on the surface of the protein, with its peptide backbone exposed to solvent. We note that while the density for both molecules is unequivocal in both chains, the density is marginally more robust for the buried molecule, suggesting that there is perhaps minor flexibility in the binding position of the exposed acinetobactin molecule.

There are no direct polar interactions between the more buried acinetobactin molecule and BauB. The DHB carbonyl oxygen interacts via water molecules with the side chain of Asp83 and the main chain amides of Ile104 and Val105. The methyl group from the heterocycle projects into a hydrophobic pocket formed byTyr84, Val264, and Tyr301. In contrast, the more exposed acinetobactin molecule makes two polar interactions with the 
protein. The isoxazolidinone carbonyl oxygen interacts with the side chain of $\operatorname{Arg} 217$ through a water molecule and a hydrogen bond is formed between one catechol oxygen and the side chain of Tyr301.

The bound holo-siderophore is presumed to exist as the monoanion [( $\left.\mathrm{Acb})_{2} \mathrm{Fe}\right]^{-}$, possibly charge stabilized by a cationic Arg217, with four catecholate oxygens balanced by iron(III) and neutral donation of two imidazole nitrogens to complete the octahedral metal coordination sphere. Interestingly, in both chains, the side chain of Arg282 from a symmetry-related molecule is directed into the binding pocket and interacts with one of the catechol oxygens of the buried acinetobactin molecule to further stabilize the [(Acb $\left.)_{2} \mathrm{Fe}\right]^{-}$ monoanion. It is also noteworthy that the contribution of the $\mathrm{N}$-terminal globular domain is largely hydrophobic while the residues from the C-terminal domain are much more polar in nature. In particular, Arg217, Arg261, Tyr301, and potentially His239 all reside on the Cterminal domain.

Conformational flexibility and electrostatics are both important factors contributing to the high-affinity association of siderophore binding proteins with substrate metal complexes as well as interfacing with membrane-embedded permeases that gate and drive the influx of substrates to the cytoplasm. Based on the ability of $A$. baumannii to utilize both isomeric forms of acinetobactin, $\mathbf{1}$ and $\mathbf{2}$, a high degree of substrate plasticity for BauB is anticipated. ${ }^{24}$ In the homologous SBP CeuE from Campylobacter jejuni, the side chains of His227 and Tyr288, the homolog of BauB His239 and Tyr301, form direct interactions with the ferric ion in complexes with catechol-based tetradentate ligands derived from enterobactin fragments that do not complete the octahedral coordination (Figure S5). ${ }^{50,51}$ The conservation of His 239 and Tyr301 suggests that BauB is poised to capture ferric complexes with alternate siderophore ligands that do not completely coordinate the central ion, perhaps endowing $A$. baumannii with the ability to interact with other siderophores that it encounters in the environment. Of note, His 239 in BauB is positioned on a poorly ordered loop, suggesting an ability to adopt distinct conformations in the presence of different ligands (Figure S5).

Siderophore binding proteins with strongly anionic or cationic binding pockets are often found to counterbalance the net charge of cognate siderophore-iron complexes (anionic staphyloferrin A-Fe, cationic HtsA; anionic staphyloferrin B-Fe, cationic SirA). ${ }^{52}, 53$ The base of the Bacillus subtilis FeuA SBP, which has been structurally characterized bound to ferric enterobactin, shows a strikingly basic binding pocket with two lysine and two arginine sidechains directed into the binding pocket. ${ }^{54}$ The 1:1 tricatecholate enterobactin-Fe complex presumably binds as the trianion $[\mathrm{EntFe}]^{3-}$, charge stabilized by protonated Lys and Arg residues lining the FeuA siderophore binding pocket. These residues are not conserved in BauB, as has also been observed with other catechol binding SBPs. ${ }^{54}$ In contrast, the base of the pocket in BauB is mostly hydrophobic. In particular, Tyr84 and Pro124 in BauB are replaced by lysine residues in FeuA, while Leu203 is replaced by an arginine.

Like BauB, the siderophore binding pocket of FhuD2 does not exhibit the basic nature of FeuA. ${ }^{55}, 56$ However, the pockets of BauB and FhuD2 are quite different in composition with BauB being much more hydrophobic. The pocket of FhuD2 is polar, with multiple 
tyrosine, threonine, and tryptophan residues donating hydrogen bonding capabilities to interact with polar hydroxamate ligands such as ferrioxamine $\mathrm{B}$. These polar residues are distributed across the binding pocket from both the $\mathrm{N}$ - and $\mathrm{C}$-terminal globular domains.

\section{DFT Calculations of Acinetobactin ${ }_{2} \mathrm{Fe}^{3+}$ complex.}

We next explored the ligand complexes using density functional theory (DFT) calculations to assess the stability of the observed 2:1 complex with other potential isomers. The metal coordination mode and ligand:metal stoichiometry observed in the BauB-bound $\mathrm{Acb}_{2} \mathrm{Fe}$ complex is consistent with reported solution-phase fluorescence and optical absorbance spectroscopic titrations. ${ }^{24,27,28}$ The presence of stable metal coordinate bonds through two bidentate catecholates is consistent with the broad metal-ligand charge transfer bands at $\sim 570 \mathrm{~nm}$ in the optical absorbance spectrum. ${ }^{24,27,28}$ The cis- $\left.^{-} \mathrm{Acb}_{2} \mathrm{Fe}\right]^{-}$isomeric form present in the BauB substrate binding site represents one of many possible geometric and optical isomeric forms of the metal complex invoking metal coordinate bonds with the catecholate and imidazole moieties. The distorted octahedron formed at the iron(III) coordination sphere is isomeric with $\mathrm{ML}^{\mathrm{a}}{ }_{4} \mathrm{~L}_{2}{ }_{2}$ octahedral complexes where $\mathrm{L}^{\mathrm{a}}$ represents the catecholate oxygens and $\mathrm{L}^{\mathrm{b}}$ represents the imidazole nitrogens. For $\mathrm{ML}_{4}^{\mathrm{a}} \mathrm{L}^{\mathrm{b}}{ }_{2}$ complexes, two isomers, cis and trans, are possible. The cis- $\left[\mathrm{FeO}_{4} \mathrm{~N}_{2}\right]^{-}$orientation is accommodated by the BauB substrate binding pocket; thus, we will refer to the holo-siderophore as cis$\left[\mathrm{Acb}_{2} \mathrm{Fe}\right]^{-}$as the apparent BauB substrate.

The siderophore, anguibactin, produced by the fish pathogen Vibrio anguillarum is structurally similar the pre-acinetobactin (2) isomeric form with the methyloxazoline replaced by a thiazoline heterocycle. Anguibactin has been crystallized as the gallium(III) chelate in a $\mathrm{Ga}_{2}$ Anguibactin 2 stoichimetry. ${ }^{57}$ The $\mathrm{Ga}_{2}$ Anguibactin 2 structure is bioctahedral with bridging methanols in the Ga coordination sphere. There are many potential stoichiometric, geometric, and optical isomers for the acinetobactin-Fe complex, including structures related to the $\mathrm{Ga}_{2}$ Anguibactin ${ }_{2}$ structure, that may be present in solution. It is likely that $\mathrm{BauB}$ selectively recognizes the $c i s-\left[\mathrm{Acb}_{2} \mathrm{Fe}\right]^{-}$isomeric form. We modeled this isomeric form and others using DFT calculations and found that structures closely related to the cis-[Acb $\left.{ }_{2} \mathrm{Fe}\right]^{-}$are predicted to be stable in the gas phase (Figure 4; Supplementary Information, Figure S6). Even if $c i s-\left[\mathrm{Acb}_{2} \mathrm{Fe}\right]^{-}$is not prevalent in solution, it is possible that upon BauB binding the coordination mode is shifted to the experimentally observed cis$\left[\mathrm{Acb}_{2} \mathrm{Fe}\right]^{-}$. Such protein-induced shifting of metal coordination mode has been observed for the binding of holo-vibrobactin by human siderocalin, where the phenolate-oxazoline bidentate ligand coordination mode predominates in solution while the bidentate catecholate mode is favored in the siderocalin substrate binding pocket. ${ }^{58}$ Binding of apo-siderophores by periplasmic siderophore binding proteins and siderocalins has also been observed. In the case of human siderocalin, the substrate binding pocket can serve as a template for small dietary catechols to preorganize the ligands for iron sequestration as a strategy for nutritional immunity, and possibly iron acquisition. ${ }^{59}$

\section{Binding Studies of Acinetobactin ${ }_{2} \mathrm{Fe}^{3+}$ to BauB.}

To investigate the substrate binding specificity of BauB in solution we performed intrinsic Trp fluorescence quenching studies in the presence of apo-and holo-acinetobactin. Both 
forms of the siderophore were bound by BauB with nanomolar affinity, with a slight preference for the holo-siderophore (apparent $K_{\mathrm{d}}=160 \pm 80 \mathrm{nM}$ ) over the apo-siderophore (apparent $K_{\mathrm{d}}=300 \pm 100 \mathrm{nM}$ ) (Table 2). We probed key structural features of acinetobactin required for molecular recognition by $\mathrm{BauB}$, including the site and degree of hydroxylation on the phenyl ring and the presence of the imidazole heterocycle. ${ }^{27}$

BauB binds both the apo and holo forms of acinetobactin. We therefore chose to use only apo siderophore variants for comparison since some structural modifications result in loss of iron chelation. We also note that we have obtained diffraction quality crystals of BauB from crystals that were grown in the presence of apo-acinetobactin. We were able to fully resolve the structure, but electron density of the substrate binding pocket did not show full occupancy of an acinetobactin molecule. A peak of ambiguous electron density was observed in the binding pocket that could fit the central isoxazolidinone ring with no density for the flanking DHB and histamine groups. Whether this represents limited occupancy or movement of the aromatic groups in the absence of iron is unclear but it is intriguing to consider the possibility that the apo-acinetobactin core is bound flexibly with iron binding subsequently ordering the histamine and DHB rings.

All of the siderophores caused dose-dependent fluorescence quenching and curve fitting provided apparent $K_{\mathrm{d}}$ values ranging from $160-520 \mathrm{nM}$, with no statistical significance between analogs (Table 2, Figure S7). It appears that BauB can bind promiscuously to the acinetobactin scaffold, which is consistent with related periplasmic siderophore binding proteins. ${ }^{10-13}$ Presumably, the outer membrane receptor BauA imparts selectivity for acinetobactin import mitigating the need for a second level of high specificity filtering in the periplasm (Figure S8). Although compounds 1, 1-Fe, and 3-9 all appear to bind BauB, it is less clear if they share the same binding mode or if all binding modes will lead to influx across the cytoplasmic membrane. The only strong correlation observed for the acinetobactin analogs is that the ability to form a stable complex with iron(III) is required for $A$. baumannii utilization (Table 2$)^{27}$.

\section{SUMMARY AND CONCLUSIONS}

We report here the structural and functional analysis of BauB, the SBP from the acinetobactin uptake pathway in $A$. baumannii. Our studies show that BauB adopts the typical Class A substrate binding fold, with a central a-helix spanning the smaller $\mathrm{N}$ - and $\mathrm{C}$ terminal domains. The ligand is bound in a central pocket between the two domains that is contributed by mostly hydrophobic residues. The structure of BauB illustrates an unusual $\left[\mathrm{Acb}_{2} \mathrm{Fe}\right]^{-1}$ complex in which two acinetobactin molecules each provide two catechol oxygens and an imidazole nitrogen to coordinate the central iron. To our knowledge, this is the first observation of a 2:1 complex of a natural ligand and iron that has been observed in a crystal structure of a siderophore binding protein.

These studies continue our on-going effort to functionally characterize the iron acquisition pathways of $A$. baumannii. The residues of BauB that interact with the ligand include hydrophobic residues that are distributed in sequence across the length of the protein and include few direct interactions with the ligand. The lack of direct interactions enables 
multiple analogs of acinetobactin to bind BauB with roughly similar affinities and may relate to the ability of bacteria to tune their iron acquisition systems to enable utilization of siderophores produced by other species.

The broad substrate binding capability of BauB presents the opportunity for exploiting the acinetobactin transport pathway to develop siderophore-based therapeutics, including antivirulence agents that inhibit the acinetobactin pathway and acinetobactin-conjugates including imaging probes for infection diagnostics and antibiotic conjugates for targeted anti-A. baumannii therapeutic agents. As noted, we have collected data on the structure of $\mathrm{BauB}$ that was crystallized in the presence of acinetobactin in the absence of iron that shows either binding to core of the siderophore or incomplete occupancy. Because of the comparable affinities of $\mathrm{BauB}$ for the apo and holo forms, we favor the former scenario in which the apo ligand binds to the hydrophobic base of the BauB pocket and the chelating groups adopt a dynamic configuration in the absence of iron. This is further supported by the similar affinity of BauB toward the acinetobactin analogs that we tested. We have additionally attempted to crystallize BauB with the oxazoline pre-Acb but have not yet been able to grow diffraction quality crystals. Continued efforts to crystallize BauB with pre-Acb or additional acinetobactin variants may provide further clues to the features that enable binding to BauB. While the histamine and isoxazolidinone are buried in one of the two acinetobactin molecules, the DHB moieties are exposed in both molecules within the $\left[\mathrm{Acb}_{2} \mathrm{Fe}\right]^{-1}$ complex and may provide a locus for attachment of relevant functional groups to achieve these alternate purposes.

\section{Supplementary Material}

Refer to Web version on PubMed Central for supplementary material.

\section{Acknowledgements.}

This work was supported in part by the National Institutes of Health Grant AI116998 (to A.M.G.) and National Science Foundation Award 1654611 to (T.A.W.). Diffraction data were collected at both the GM/CA ID-B beamline of APS and beamline 12-2 at SSRL. The GM/CA line is supported by the National Cancer Institute Grant ACB-12002, National Institute of General Medical Sciences Grant AGM-12006 under Department of Energy Contract Number DE-AC02- 06CH11357 to the National Cancer Institute (ACB-12002), and the National Institute of General Medical Sciences (AGM-12006). This research used resources of the Advanced Photon Source, a U.S. Department of Energy (DOE) Office of Science User Facility operated for the DOE Office of Science by Argonne National Laboratory under Contract No. DE-AC02-06CH11357. The Eiger 16M detector was funded by an NIHOffice of Research Infrastructure Programs, High-End Instrumentation Grant (1S10OD012289-01A1). Use of the Stanford Synchrotron Radiation Lightsource, SLAC National Accelerator Laboratory, is supported by the U.S. Department of Energy, Office of Science, Office of Basic Energy Sciences under Contract No. DE-AC0276SF00515. The SSRL Structural Molecular Biology Program is supported by the DOE Office of Biological and Environmental Research, and by the National Institutes of Health, National Institute of General Medical Sciences (including P41GM103393).

\section{REFERENCES}

[1]. Hider RC, and Kong X (2010) Chemistry and biology of siderophores, Nat Prod Rep 27, 637-657. [PubMed: 20376388]

[2]. Sandy M, and Butler A (2009) Microbial iron acquisition: marine and terrestrial siderophores, Chem Rev 109, 4580-4595. [PubMed: 19772347]

[3]. Bilitewski U, Blodgett JAV, Duhme-Klair AK, Dallavalle S, Laschat S, Routledge A, and Schobert $\mathrm{R}$ (2017) Chemical and biological aspects of nutritional immunity-perspectives for new anti- 
infectives that target iron uptake systems, Angew Chem Int Ed Engl 56, 14360-14382. [PubMed: 28439959]

[4]. Miethke M, and Marahiel MA (2007) Siderophore-based iron acquisition and pathogen control, Microbiol Mol Biol Rev 71, 413-451. [PubMed: 17804665]

[5]. Lamb AL (2015) Breaking a pathogen's iron will: Inhibiting siderophore production as an antimicrobial strategy, Biochim Biophys Acta 1854, 1054-1070. [PubMed: 25970810]

[6]. Crosa JH, and Walsh CT (2002) Genetics and assembly line enzymology of siderophore biosynthesis in bacteria, Microbiol Mol Biol Rev 66, 223-249. [PubMed: 12040125]

[7]. Gulick AM (2016) Structural insight into the necessary conformational changes of modular nonribosomal peptide synthetases, Curr Opin Chem Biol 35, 89-96. [PubMed: 27676239]

[8]. Kadi N, Oves-Costales D, Barona-Gomez F, and Challis GL (2007) A new family of ATPdependent oligomerization-macrocyclization biocatalysts, Nat Chem Biol 3, 652-656. [PubMed: 17704771]

[9]. Oves-Costales D, Kadi N, and Challis GL (2009) The long-overlooked enzymology of a nonribosomal peptide synthetase-independent pathway for virulence-conferring siderophore biosynthesis, Chem Commun (Camb), 6530-6541. [PubMed: 19865642]

[10]. Chu BC, Garcia-Herrero A, Johanson TH, Krewulak KD, Lau CK, Peacock RS, Slavinskaya Z, and Vogel HJ (2010) Siderophore uptake in bacteria and the battle for iron with the host; a bird's eye view, Biometals 23, 601-611. [PubMed: 20596754]

[11]. Lewinson O, and Livnat-Levanon N (2017) Mechanism of Action of ABC Importers: Conservation, Divergence, and Physiological Adaptations, J Mol Biol 429, 606-619. [PubMed: 28104364]

[12]. Tanaka KJ, Song S, Mason K, and Pinkett HW (2018) Selective substrate uptake: The role of ATP-binding cassette (ABC) importers in pathogenesis, Biochim Biophys Acta 1860, 868-877.

[13]. Yang M, Livnat Levanon N, Acar B, Aykac Fas B, Masrati G, Rose J, Ben-Tal N, Haliloglu T, Zhao Y, and Lewinson O (2018) Single-molecule probing of the conformational homogeneity of the ABC transporter BtuCD, Nat Chem Biol 14, 715-722. [PubMed: 29915236]

[14]. Locher KP (2016) Mechanistic diversity in ATP-binding cassette (ABC) transporters, Nat Struct Mol Biol 23, 487-493. [PubMed: 27273632]

[15]. Berntsson RP, Smits SH, Schmitt L, Slotboom DJ, and Poolman B (2010) A structural classification of substrate-binding proteins, FEBS Lett 584, 2606-2617. [PubMed: 20412802]

[16]. Scheepers GH, Lycklama a Nijeholt JA, and Poolman B (2016) An updated structural classification of substrate-binding proteins, FEBS Lett 590, 4393-4401. [PubMed: 27714801]

[17]. Dorsey CW, Tolmasky ME, Crosa JH, and Actis LA (2003) Genetic organization of an Acinetobacter baumannii chromosomal region harbouring genes related to siderophore biosynthesis and transport, Microbiology 149, 1227-1238. [PubMed: 12724384]

[18]. Dorsey CW, Tomaras AP, Connerly PL, Tolmasky ME, Crosa JH, and Actis LA (2004) The siderophore-mediated iron acquisition systems of Acinetobacter baumannii ATCC 19606 and Vibrio anguillarum 775 are structurally and functionally related, Microbiology 150, 3657-3667. [PubMed: 15528653]

[19]. Proschak A, Lubuta P, Grun P, Lohr F, Wilharm G, De Berardinis V, and Bode HB (2013) Structure and biosynthesis of fimsbactins A-F, siderophores from Acinetobacter baumannii and Acinetobacter baylyi, Chembiochem 14, 633-638. [PubMed: 23456955]

[20]. Penwell WF, DeGrace N, Tentarelli S, Gauthier L, Gilbert CM, Arivett BA, Miller AA, DurandReville TF, Joubran C, and Actis LA (2015) Discovery and Characterization of New Hydroxamate Siderophores, Baumannoferrin A and B, produced by Acinetobacter baumannii, Chembiochem 16, 1896-1904. [PubMed: 26235845]

[21]. Chen L, Zheng D, Liu B, Yang J, and Jin Q (2016) VFDB 2016: hierarchical and refined dataset for big data analysis--10 years on, Nucleic Acids Res 44, D694-697. [PubMed: 26578559]

[22]. Vallenet D, Nordmann P, Barbe V, Poirel L, Mangenot S, Bataille E, Dossat C, Gas S, Kreimeyer A, Lenoble P, Oztas S, Poulain J, Segurens B, Robert C, Abergel C, Claverie JM, Raoult D, Medigue C, Weissenbach J, and Cruveiller S (2008) Comparative analysis of Acinetobacters: three genomes for three lifestyles, PLoS One 3, e1805. [PubMed: 18350144] 
[23]. Gaddy JA, Arivett BA, McConnell MJ, Lopez-Rojas R, Pachon J, and Actis LA (2012) Role of acinetobactin-mediated iron acquisition functions in the interaction of Acinetobacter baumannii strain ATCC 19606T with human lung epithelial cells, Galleria mellonella caterpillars, and mice, Infect Immun 80, 1015-1024. [PubMed: 22232188]

[24]. Shapiro JA, and Wencewicz TA (2016) Acinetobactin Isomerization Enables Adaptive Iron Acquisition in Acinetobacter baumannii through pH-Triggered Siderophore Swapping, ACS Infect Dis 2, 157-168. [PubMed: 27624967]

[25]. Neres J, Engelhart CA, Drake EJ, Wilson DJ, Fu P, Boshoff HI, Barry CE 3rd, Gulick AM, and Aldrich CC (2013) Non-nucleoside inhibitors of BasE, an adenylating enzyme in the siderophore biosynthetic pathway of the opportunistic pathogen Acinetobacter baumannii, J Med Chem 56, 2385-2405. [PubMed: 23437866]

[26]. Drake EJ, Duckworth BP, Neres J, Aldrich CC, and Gulick AM (2010) Biochemical and structural characterization of bisubstrate inhibitors of BasE, the self-standing nonribosomal peptide synthetase adenylate-forming enzyme of acinetobactin synthesis, Biochemistry 49, 92929305. [PubMed: 20853905]

[27]. Shapiro JA, and Wencewicz TA (2017) Structure-function studies of acinetobactin analogs, Metallomics 9, 463-470. [PubMed: 28440833]

[28]. Bohac TJ, Shapiro JA, and Wencewicz TA (2017) Rigid Oxazole Acinetobactin Analog Blocks Siderophore Cycling in Acinetobacter baumannii, ACS Infect Dis 3, 802-806. [PubMed: 28991447]

[29]. Nielsen H (2017) Predicting Secretory Proteins with SignalP, Methods Mol Biol 1611, 59-73. [PubMed: 28451972]

[30]. Kall L, Krogh A, and Sonnhammer EL (2007) Advantages of combined transmembrane topology and signal peptide prediction--the Phobius web server, Nucleic Acids Res 35, W429-432. [PubMed: 17483518]

[31]. Braun v., and Wu HC. (1994) Lipoproteins, structure, function, biosynthesis and model for protein export, In Compr New. Biochem. (Ghuysen JM, and Hakenbeck R, Eds.), pp 319-341, Elsevier.

[32]. Juncker AS, Willenbrock H, Von Heijne G, Brunak S, Nielsen H, and Krogh A (2003) Prediction of lipoprotein signal peptides in Gram-negative bacteria, Protein Sci 12, 1652-1662. [PubMed: 12876315]

[33]. Seydel A, Gounon P, and Pugsley AP (1999) Testing the '+2 rule' for lipoprotein sorting in the Escherichia coli cell envelope with a new genetic selection, Mol Microbiol 34, 810-821. [PubMed: 10564520]

[34]. Yamaguchi K, Yu F, and Inouye M (1988) A single amino acid determinant of the membrane localization of lipoproteins in E. coli, Cell 53, 423-432. [PubMed: 3284654]

[35]. Zawadzka AM, Kim Y, Maltseva N, Nichiporuk R, Fan Y, Joachimiak A, and Raymond KN (2009) Characterization of a Bacillus subtilis transporter for petrobactin, an anthrax stealth siderophore, Proc Natl Acad Sci U S A 106, 21854-21859. [PubMed: 19955416]

[36]. Battye TG, Kontogiannis L, Johnson O, Powell HR, and Leslie AG (2011) iMOSFLM: a new graphical interface for diffraction-image processing with MOSFLM, Acta Crystallogr D Biol Crystallogr 67, 271-281. [PubMed: 21460445]

[37]. McCoy AJ, Grosse-Kunstleve RW, Adams PD, Winn MD, Storoni LC, and Read RJ (2007) Phaser crystallographic software, J. Appl. Crystallography 40, 658-674.

[38]. Terwilliger TC, Grosse-Kunstleve RW, Afonine PV, Moriarty NW, Zwart PH, Hung LW, Read RJ, and Adams PD (2008) Iterative model building, structure refinement and density modification with the PHENIX AutoBuild wizard, Acta Crystallogr D Biol Crystallogr 64, 6169. [PubMed: 18094468]

[39]. Emsley P, and Cowtan K (2004) Coot: model-building tools for molecular graphics, Acta Crystallogr D Biol Crystallogr 60, 2126-2132. [PubMed: 15572765]

[40]. Afonine PV, Grosse-Kunstleve RW, Echols N, Headd JJ, Moriarty NW, Mustyakimov M, Terwilliger TC, Urzhumtsev A, Zwart PH, and Adams PD (2012) Towards automated crystallographic structure refinement with phenix.refine, Acta Crystallogr D Biol Crystallogr 68, 352-367. [PubMed: 22505256] 
[41]. Pei J, and Grishin NV (2014) PROMALS3D: multiple protein sequence alignment enhanced with evolutionary and three-dimensional structural information, Methods Mol Biol 1079, 263-271. [PubMed: 24170408]

[42]. Pople JA, Scott AP, Wong MW, and Radom L (1993) Scaling factors for obtaining fundamental vibrational frequencies and zero-point energies from $\mathrm{Hf} / 6-31 \mathrm{G}^{*}$ and $\mathrm{Mp} 2 / 6-31 \mathrm{G}^{*}$ Harmonic Frequencies, Isr J Chem 33, 345-350.

[43]. Cossi M, Rega N, Scalmani G, and Barone V (2003) Energies, structures, and electronic properties of molecules in solution with the C-PCM solvation model, Journal of Computational Chemistry 24, 669-681. [PubMed: 12666158]

[44]. Shephard MJ, and Paddonrow MN (1995) Gas-Phase structure of the bicyclo[2.2.1]heptane (Norbornane) cation-radical - a combined ab-initio MO and density-functional study, J Phys Chem-US 99, 3101-3108.

[45]. Zhao Y, and Truhlar DG (2008) Density functionals with broad applicability in chemistry, Acc Chem Res 41, 157-167. [PubMed: 18186612]

[46]. Rydberg P, and Olsen L (2009) The accuracy of geometries for iron porphyrin complexes from density functional theory, J Phys Chem A 113, 11949-11953. [PubMed: 19663404]

[47]. Ansbacher T, Srivastava HK, Martin JM, and Shurki A (2010) Can DFT methods correctly and efficiently predict the coordination number of copper(I) complexes? A case study, Journal of computational chemistry 31, 75-83. [PubMed: 19412907]

[48]. Weigend F, and Ahlrichs R (2005) Balanced basis sets of split valence, triple zeta valence and quadruple zeta valence quality for $\mathrm{H}$ to $\mathrm{Rn}$ : Design and assessment of accuracy, Phys Chem Chem Phys 7, 3297-3305. [PubMed: 16240044]

[49]. Holm L, and Laakso LM (2016) Dali server update, Nucleic Acids Res 44, W351-355. [PubMed: 27131377]

[50]. Raines DJ, Moroz OV, Wilson KS, and Duhme-Klair AK (2013) Interactions of a periplasmic binding protein with a tetradentate siderophore mimic, Angew Chem Int Ed Engl 52, 4595-4598. [PubMed: 23512642]

[51]. Raines DJ, Moroz OV, Blagova EV, Turkenburg JP, Wilson KS, and Duhme-Klair AK (2016) Bacteria in an intense competition for iron: Key component of the Campylobacter jejuni iron uptake system scavenges enterobactin hydrolysis product, Proc Natl Acad Sci U S A 113, 58505855. [PubMed: 27162326]

[52]. Grigg JC, Cooper JD, Cheung J, Heinrichs DE, and Murphy ME (2010) The Staphylococcus aureus siderophore receptor HtsA undergoes localized conformational changes to enclose staphyloferrin A in an arginine-rich binding pocket, J Biol Chem 285, 11162-11171. [PubMed: 20147287]

[53]. Grigg JC, Cheung J, Heinrichs DE, and Murphy ME (2010) Specificity of Staphyloferrin B recognition by the SirA receptor from Staphylococcus aureus, J Biol Chem 285, 34579-34588. [PubMed: 20810662]

[54]. Peuckert F, Ramos-Vega AL, Miethke M, Schworer CJ, Albrecht AG, Oberthur M, and Marahiel MA (2011) The siderophore binding protein FeuA shows limited promiscuity toward exogenous triscatecholates, Chem Biol 18, 907-919. [PubMed: 21802011]

[55]. Endicott NP, Lee E, and Wencewicz TA (2017) Structural Basis for Xenosiderophore Utilization by the Human Pathogen Staphylococcus aureus, ACS Infect Dis 3, 542-553. [PubMed: 28505405]

[56]. Podkowa KJ, Briere LA, Heinrichs DE, and Shilton BH (2014) Crystal and solution structure analysis of FhuD2 from Staphylococcus aureus in multiple unliganded conformations and bound to ferrioxamine-B, Biochemistry 53, 2017-2031. [PubMed: 24606332]

[57]. Hossain MB, Jalal MAF, and van der Helm D (1998) Gallium-complex of anguibactin, a siderophore from fish pathogen Vibrio anguillarum, J Chem Crystallogr 28, 57-60.

[58]. Allred BE, Correnti C, Clifton MC, Strong RK, and Raymond KN (2013) Siderocalin outwits the coordination chemistry of vibriobactin, a siderophore of Vibrio cholerae, ACS Chem Biol 8, 1882-1887. [PubMed: 23755875] 
[59]. Shields-Cutler RR, Crowley JR, Miller CD, Stapleton AE, Cui W, and Henderson JP (2016) Human Metabolome-derived Cofactors Are Required for the Antibacterial Activity of Siderocalin in Urine, J Biol Chem 291, 25901-25910. [PubMed: 27780864] 


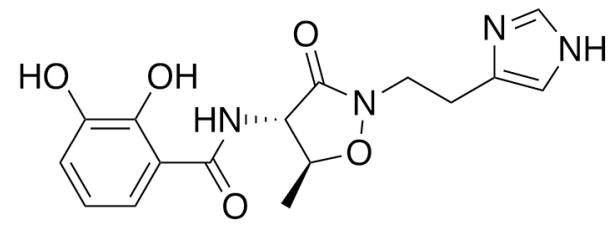

Acinetobactin (1)

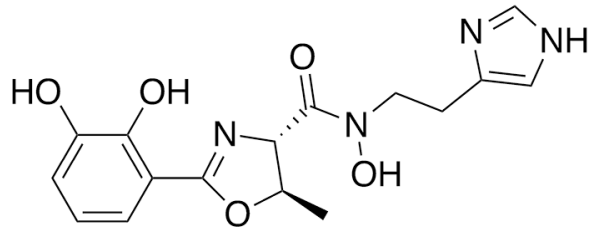

Pre-acinetobactin (2)

Figure 1.

Chemical structures of acinetobactin (1) and pre-acinetobactin (2) from A. baumannii. 


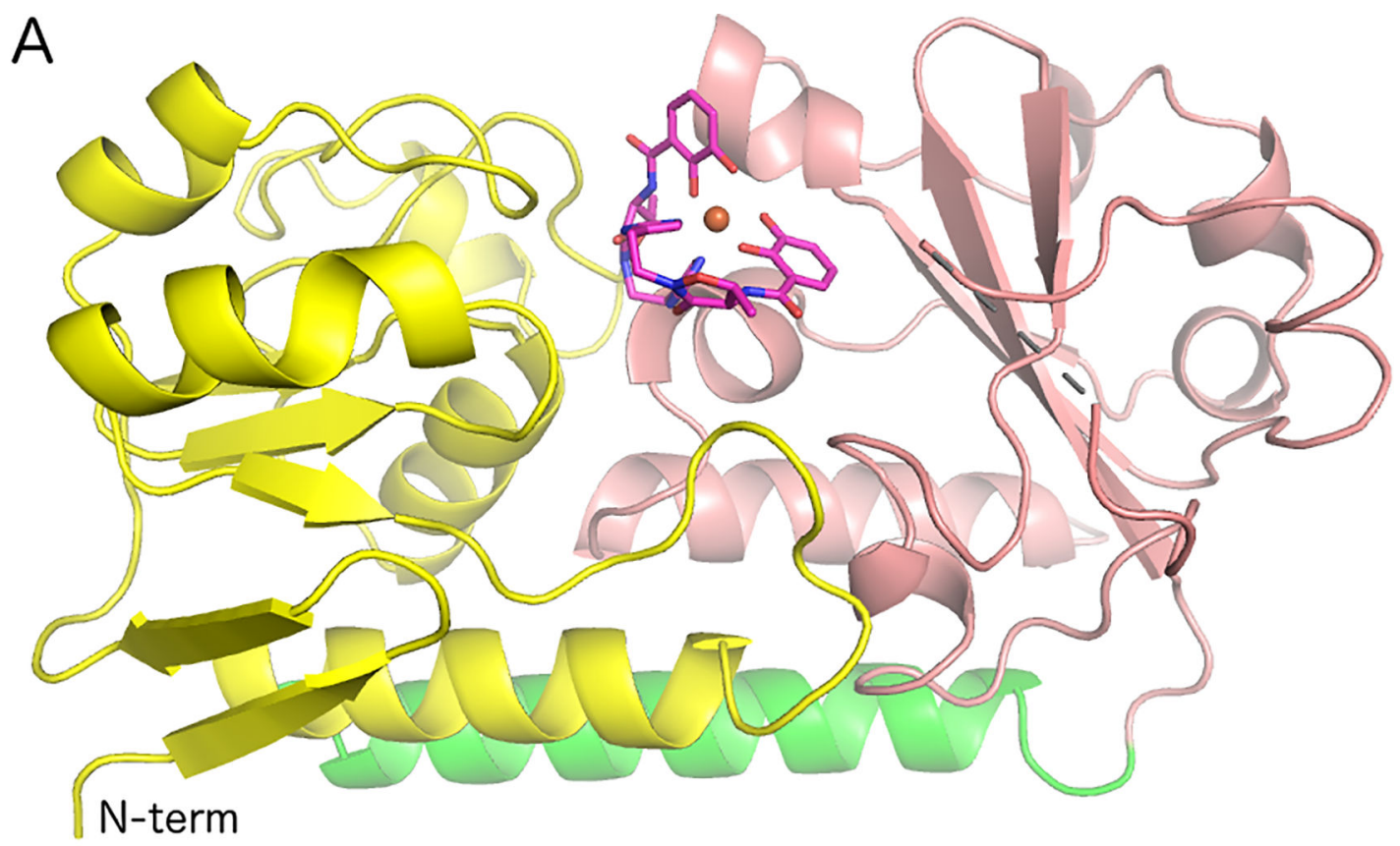

B

Figure 2.

Ribbon representation of BauB bound to the Acb2 $\bullet$ Fe complex. (A) Highlight of the two domains and the $a$ helix that joins them (N-domain, yellow; $\mathrm{C}$-domain pink; $a$ helix green) (B) Orthogonal view, rotated approximately $90^{\circ}$ around the horizontal axis. The single gap in the protein between residues 235 and 239 is indicated with the dashed line. The Acb molecules are shown in stick representation with magenta carbon, red oxygen, and blue nitrogen atoms. 

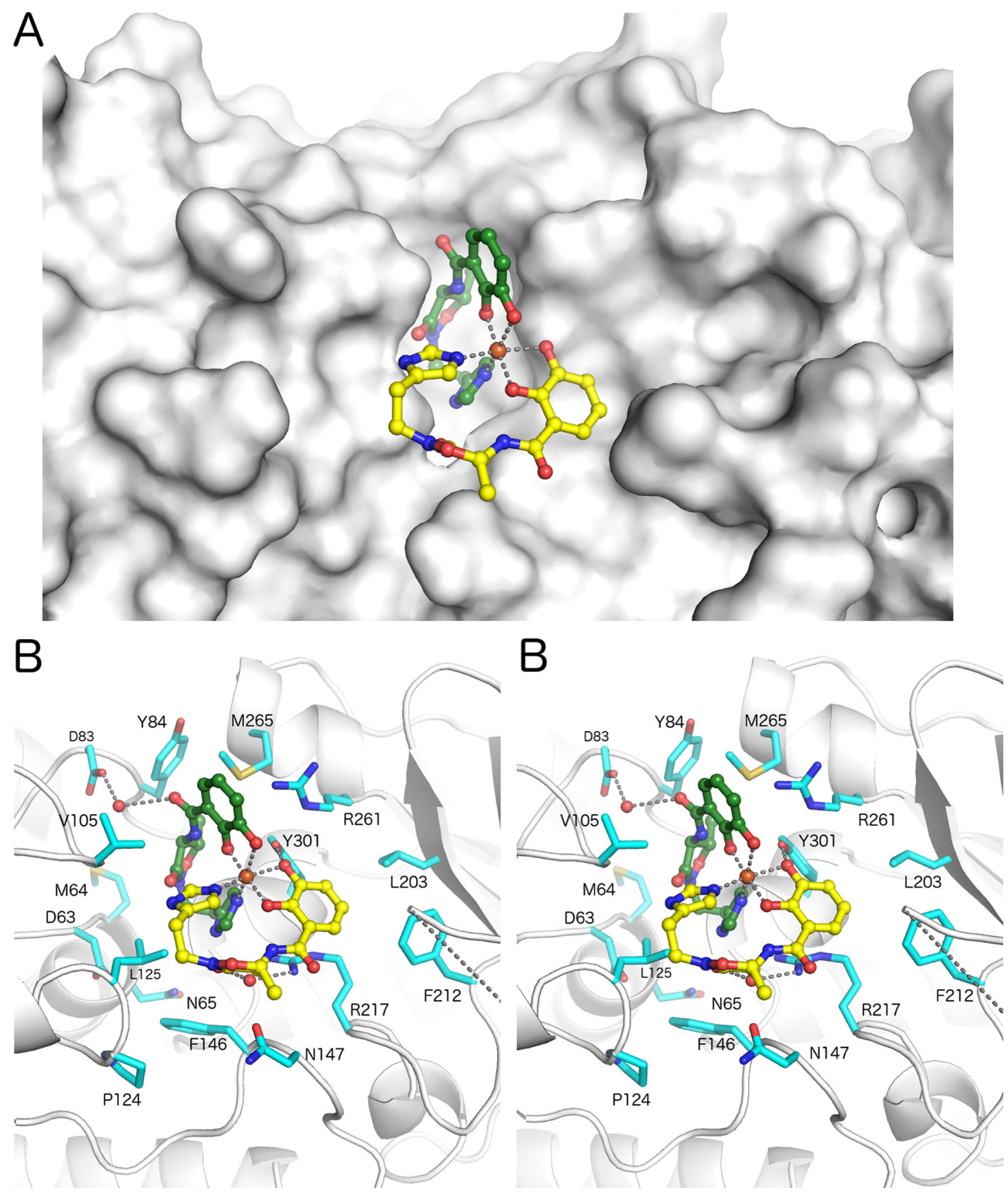

Figure 3.

Siderophore binding pocket in BauB. (A) The $\mathrm{Acb}_{2} \mathrm{Fe}$ ligand is shown with buried (green) and exposed (yellow) acinetobactin molecules with a surface representation of BauB. (B) Stereorepresentation of the residues that form the binding pocket. 

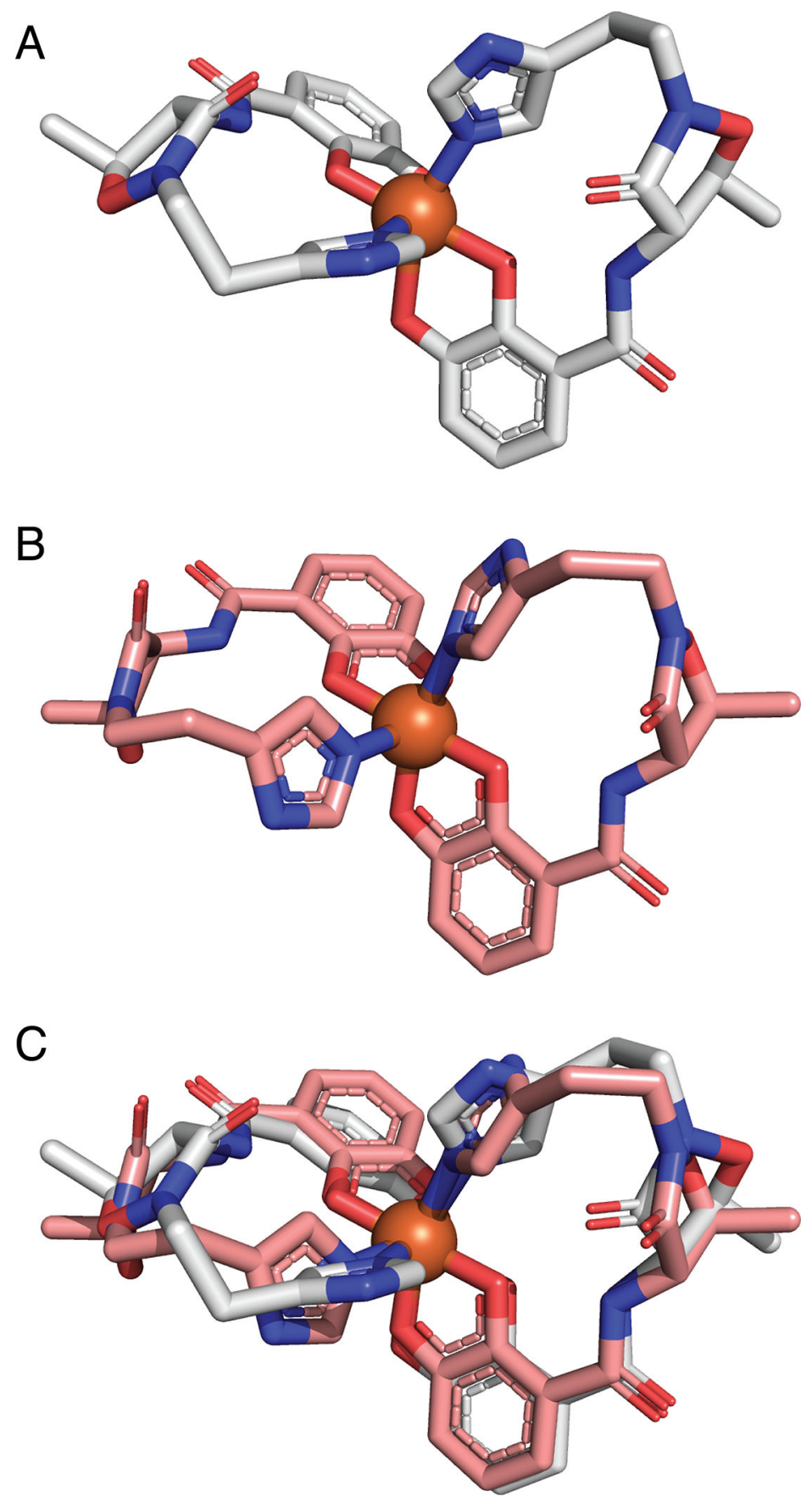

Figure 4.

Structures of $(\mathrm{Acb})_{2} \mathrm{Fe}$ from (A) experimental fitting into the electron density of the BauB substrate binding pocket and (B) DFT calculated lowest energy structure for the monoanionic $\left[(\mathrm{Acb})_{2} \mathrm{Fe}\right]^{-}$metal complex (PBE0/Def2-SVP; PBE0/Def2-TZVP; PBE1PBE $=$ PBE0 in Gaussian). (C) Overlay of (Acb) $)_{2} \mathrm{Fe}$ structures from experimental (grey) and DFT (salmon) modeling. 
Table 1.

Data collection and refinement statistics

\begin{tabular}{|c|c|}
\hline & BauB-Acb $2-\mathrm{Fe}^{3+}$ \\
\hline PDB ID & 6MFL \\
\hline \multicolumn{2}{|l|}{ Data Collection } \\
\hline Resolution range $(\AA)$ & $68-1.9(1.94-1.90)$ \\
\hline Space group & $\mathrm{P} 2_{1}$ \\
\hline Unit cell a, b, c (̊̊) $a, \beta, \gamma\left(^{\circ}\right)$ & $37.8,137.1,56.2,90,90.0,90$ \\
\hline Number Crystals & 2 \\
\hline Total Observations & $347119(22375)$ \\
\hline Unique reflections & 44580 (2908) \\
\hline Multiplicity & 7.8 \\
\hline Completeness (\%) & $99.2(99.6)$ \\
\hline Mean I/sigma(I) & $11.6(5.6)$ \\
\hline $\mathrm{R}_{\text {MERGE }}$ & $0.178(0.570)$ \\
\hline $\mathrm{R}_{\text {MEAS }}$ & $0.204(0.662)$ \\
\hline $\mathrm{CC} 1 / 2$ & $0.970(0.679)$ \\
\hline \multicolumn{2}{|l|}{ Structure Refinement } \\
\hline Reflections used in refinement & 44255 \\
\hline Reflections used for $\mathrm{R}_{\mathrm{FREE}}$ & 2140 \\
\hline $\mathrm{R}_{\text {WORK }}$ & 16.9 \\
\hline $\mathrm{R}_{\mathrm{FREE}}$ & 21.4 \\
\hline \multicolumn{2}{|l|}{ Number of non-hydrogen atoms } \\
\hline macromolecules & 4928 \\
\hline ligands & 102 \\
\hline solvent & 372 \\
\hline Wilson B-factor $\left(\AA^{2}\right)$ & 13.8 \\
\hline \multicolumn{2}{|l|}{ Average B-factor $\left(\AA^{2}\right)$} \\
\hline Chain A (main, side) & $14.8,20.5$ \\
\hline Chain B (main, side) & $13.9,20.4$ \\
\hline $\mathrm{Acb}_{2} \mathrm{Fe}$ & 18.0 \\
\hline Water & 23.3 \\
\hline Ethylene glycol & 34.7 \\
\hline RMSD bond lengths ( $\AA$ ) & 0.01 \\
\hline RMSD bond angles $\left({ }^{\circ}\right)$ & 1.12 \\
\hline \multicolumn{2}{|l|}{ Ramachandran analysis } \\
\hline favored $(\%)$ & 97.0 \\
\hline allowed (\%) & 3.0 \\
\hline outliers (\%) & 0.0 \\
\hline Rotamer outliers (\%) & 0.8 \\
\hline MolProbity Clashscore & 2.9 \\
\hline
\end{tabular}

Biochemistry. Author manuscript; available in PMC 2019 December 04. 
Table 2.

Structure of acinetobactin analogs and associated properties.

\begin{tabular}{lccc}
\hline & & & \\
& & & \\
\end{tabular}

${ }^{1}$ Results from CAS assay and $A$. baumannii growth promotion were reported previously. ${ }^{27}$ For the CAS assay, + indicates a positive result correlating to Fe binding and a - indicates no apparent Fe binding. For the growth promotion of $A$. baumannii, a $+/++/+++$ indicates positive promotion of growth with more ' + ' symbols correlating to more relative growth.

2 Experiments were performed in duplicate as independent trials. Error represents standard deviation from the mean.p

3 NA $=$ not applicable. 